\title{
Refractive index sensing utilizing a cw photonic crystal nanolaser and its array configuration
}

\author{
Shota Kita ${ }^{1,2}$, Kengo Nozaki ${ }^{1,2}$ and Toshihiko Baba ${ }^{1,2}$ \\ ${ }^{1}$ Yokohama National University, Department of Electrical and Computer Engineering, \\ 79-5 Tokiwadai, Hodogayaku, Yokohama 240-8501, Japan \\ ${ }^{2}$ Japan and Core Research for Evolutional Science and Technology (CREST), \\ Japan Science Technology (JST) Agency, \\ 5 Sanbancho, Chiyodaku, Tokyo 102-0075, Japan \\ E-mail: baba@ynu.ac.jp
}

\begin{abstract}
We demonstrate refractive index sensing using photonic crystal point shift nanolasers. These lasers operate continuously at room temperature by photopumping in a liquid, and exhibit a $50-\mathrm{dB}$ peak intensity over the background level and a spectral linewidth of $<26 \mathrm{pm}$, the resolution limit of the present experiment. The lasing wavelength shifts by soaking in different liquids; the wavelength to index sensitivity was 350 $\mathrm{nm} / \mathrm{RIU}$, the highest value recorded to date for nanocavity- based sensors. An index resolution of $9.0 \times 10^{-5}$ was thus confirmed, leading to an expectation of a resolution of $<10^{-6}$. We propose and demonstrate a spectrometer-free sensor based on nanolasers in an array configuration. These will be disposable sensors with very simple optical I/O. They are anticipated to be integrated with biochips and used for label-free single molecule detection.
\end{abstract}

(C)2008 Optical Society of America

OCIS codes: (140.5960) Semiconductor lasers; (230.5298) Photonic crystals

\section{References and links}

1. D. Psaltis, S. R. Quake, and C. Yang "Developing optofluidic technology through the fusion of microfluidics and optics," Nature 442, 381 - 386 (2006).

2. C. Monat, P. Domachuk and B. J. Eggleton, "Integrated optofluidics: a new river of light," Nat. Photonics 1, 106-114 (2007).

3. F. Follmer, D. Braun, A. Libchaber, M. Khoshsima, I. Teraoka, and S. Arnold, "Protein detection by optical shift of a resonant microcavity," Appl. Phys. Lett. 80, 4057-4059 (2002).

4. I. M. White, H. Zhu, J. Suter, N. M. Hanumegowda, H. Oveys, M. Zourob, and X. Fan, "Refractometric sensors for lab-on-a-chip based on optical ring resonators," IEEE Sens. J. 7, 28-35 (2007).

5. A. M. Armani, R. P. Kulkarni, S. E. Fraser, R. C. Flagan, and K. J. Vahala, "Label-free, single-molecule detection with optical microcavities," Science (2007).

6. A. N. Chryssis, S. M. Lee, S. B. Lee, S. S. Saini, and M. Dagenais, "High sensitivity evanescent field fiber Bragg grating sensor," IEEE Photon. Technol. Lett. 17, 1253-1255 (2005).

7. E. Chow, A. Grot, L. W. Mirkarimi, M. Sigalas, and G. Girolami, "Ultracompact biochemical sensor built with two-dimensional photonic crystal microcavity," Opt. Lett. 29, 1093 (2004)

8. Y. Nishijima, K. Ueno, S. Juodkazis, V. Mizeikis, H. Misawa, T. Tanimura, and K. Maeda, "Inverse silica opal photonic crystals for optical sensing applications," Opt. Express 15, 12979-12987 (2007).

9. M. R. Lee and Philippe M. Fauchet, "Two-dimensional silicon photonic crystal based biosensing platform for protein detection," Opt. Express 15, 4530-4535 (2007).

10. M. R. Lee and P. M. Fauchet, "Nanoscale microcavity sensor for single particle detection," Opt. Lett. 32, 3284-3286 (2007).

11. M. Loncar, A. Scherer, and Y. Qiu, "Photonic crystal laser sources for chemical detection," Appl. Phys. Lett. 82, 4648-4650 (2003).

12. M. L. Adams, G. A. DeRose, M. Loncar and A. Scherer, "Lithographically fabricated optical cavities for refractive index sensing," J. Vac. Sci. Technol. B 23, 3168-3173 (2005).

\#94556 - \$15.00 USD Received 4 Apr 2008; revised 10 May 2008; accepted 11 May 2008; published 20 May 2008 
13. M. L. Adams, M. Loncar, A. Scherer, and Y. Qiu, "Microfluidic integration of porous photonic crystals nanolasers for chemical sensing," IEEE J. Sel. Areas Commun. 23, 1348 -1354 (2005).

14. H. Watanabe, K. Nozaki, and T. Baba, "Very Wide Wavelength Chirping in Photonic Crystal Nanolaser," The 34th International Symposium on Compound Semiconductors, ThC P10 (2007).

15. K. Nozaki, S. Kita, and T. Baba, "Room temperature continuous wave operation and controlled spontaneous emission in ultrasmall photonic crystal nanolaser," Opt. Express 15, 7506-7514 (2007).

16. K. Nozaki and T. Baba, "Laser characteristics with ultimate-small modal volume in photonic crystal slab point-shift nanolasers,” Appl. Phys. Lett. 88, 21101 (2006).

17. F. M. De Sopra, H. P. Zappe, M. Moser, R. Hovel, H. P. Gauggel, and K. Gulden, "Near-infrared vertical-cavity surface-emitting lasers with 3-MHzlinewidth,” IEEE Photon. Technol. Lett. 11, 1533 - 1535 (1999).

\section{Introduction}

Optofluidics now lies at the forefront of synthetic/analytical chemistry and nanobiotechnology. Here, light is used for controlling and efficiently analyzing fluids, colloidal solutions, solids in a fluid, etc, in microscale devices such as labs-on-a-chip [1, 2]. Sensors are among the fundamental elements of optofluidics. They are required to be compact, cheap, disposable, and highly sensitive. Recently, optical microcavities open to air have been studied extensively for sensing. The resonant wavelength simply shifts as the environmental refractive index $n_{\text {env }}$ varies. Conversely, the index can be detected by measuring the wavelength shift via a spectrometer. Thus far, microspheres [3], microrings [4], microtoroidals [5], grating [6], and photonic crystals (PCs) $[7,8]$ have been studied as microcavities for sensing. In particular, a nanocavity in the two-dimensional (2D) PC slab confines light into an ultrasmall volume of the order of optical wavelength $\left(\sim(\lambda / n)^{3}\right.$, where $\lambda$ is the resonant wavelength in vacuum and $n$ is the index of the slab). It provides not only a high spatial resolution but also a high index resolution due to its high $Q$ (narrow spectral linewidth). Thus, it enables analysis of even ultrasmall aliquots of liquids $(1 \mathrm{fl} \sim 1 \mathrm{fg})$. It can detect the molecular size when the protein monolayer is fixed adjacent to the nanocavity [9]. Label-free single molecule detection was also demonstrated [10]. These studies used passive cavities, which generally require a wideband light source, polarization controller and high-resolution spectrometer as well as high-precision optical input/output (I/O).

In order to simplify the measurement system and reduce the cost, a sensor utilizing point defect nanolasers on an active PC slab has been reported [11 - 13]. Its principle is the same as that for passive devices: the lasing wavelength shifts with the index. But active devices are advantageous because they are remotely operated by photopumping, and sensing is performed by detecting the laser light via a simple optical setup. Thus, we can neglect the external light source and high-precision optical I/O for passive devices. A critical problem with active devices is that continuous-wave (cw) operation is not obtained, but rather, only pulsed operation. When a PC nanolaser is operated by pulsed photopumping, spectral broadening often occurs due to thermal chirping [14]. It broadens the spectral linewidth to $>10 \mathrm{~nm}$ in the widest case. Thus, even if a high index sensitivity $\Delta \lambda / \Delta n_{\text {env }}$ of $250 \mathrm{~nm} / \mathrm{RIU}$ [12] is used, the index resolution is limited to an order of $10^{-3}$ by the spectral linewidth. This is far inferior to typical values in passive devices $\left(10^{-5}-10^{-6}\right)$.

In this paper, we demonstrate higher-resolution sensing using a cw PC nanolaser, in which the thermal chirping is suppressed and a much narrower linewidth is obtained. We have already achieved room-temperature $\mathrm{cw}$ operation in a point shift PC nanolaser [15]. We call this a H0 nanolaser as it has no missing airholes, but consists of only a shift in two airholes in the PC slab. We show the stable narrow spectral characteristics of the nanolaser in liquids and a record high sensitivity of $350 \mathrm{~nm} / \mathrm{RIU}$, resulting in an index resolution of $9.0 \times 10^{-5}$ (potentially $<10^{-6}$ ). Besides, we propose a spectrometer-free index sensor based on a nanolaser array. Here, many nanolasers whose lasing wavelengths are slightly different from 
each other are operated simultaneously, and their near field pattern (NFP) is observed through a step-like or delta-function-like bandpass filter (BPF). The wavelength shifts and the NFP changes with $n_{\text {env }}$. In this paper, we present the first demonstration of such an operation.

\section{Sensing characteristics of cw PC nanolasers}

The H0 nanocavity consists of the lateral shift $s_{\mathrm{x}}$ of two lattice points in a triangular lattice PC slab whose design parameters are the lattice constant $a$ and the airhole diameter $2 r$. This nanocavity maintains the monopole mode and the dipole mode, having one and two primary antinodes, respectively, in the magnetic field component $H_{\mathrm{z}}$ of the modal standing wave. In particular, the monopole mode exhibits an ultrasmall modal volume $V_{\mathrm{m}}$ of less than $0.15(\lambda / n)^{3}$ and a $Q$ factor of higher than $10^{5}$, giving rise to high performance lasing. The dipole mode exhibits a slightly larger $V_{\mathrm{m}}$ of $0.21(\lambda / n)^{3}$ and a lower $Q$ of $10^{3}-10^{4}$. But still lasing is obtained because this $Q$ is not necessarily dominant for the total $Q$ affected by parasitic losses such as free carrier absorption and light scattering by disordering in the fabricated devices. We experimentally identified these modes [16], and observed room-temperature cw lasing with an effective threshold power $P_{\text {th }}$ of $1.2 \mu \mathrm{W}$ in the monopole mode [15]. In the present study, we also introduce the shift $s_{\mathrm{y}}$ of the other two lattice points located adjacent to those for $s_{\mathrm{x}}$. The resonant wavelength and $Q$ can be flexibly controlled by varying $s_{\mathrm{x}}$ and $s_{\mathrm{y}}$. This is effective for the optimization of the nanolaser array, which will be discussed in the next section.

In this experiment, we first optimized the wafer structure. We prepared the following two epiwafers: GaInAsP single-quantum-well (SQW) wafer with a total thickness of the active layer including separate-confinement-heterostructure layers $d$ of $180 \mathrm{~nm}$, and GaInAsP five multi-quantum-well (MQW) wafers with $d=240 \mathrm{~nm}$. The photoluminescence peak of both wafers was centered at $1.55 \mu \mathrm{m}$. Assuming an air-membrane structure, we calculated $\Delta n_{\text {mode }} / \Delta n_{\text {env }}$ for these wafers, where $n_{\text {mode }}$ is the equivalent modal index. The SQW wafer was found to give a 1.8-fold higher value than that of the MQW wafer. Because of the thinner $d$, the evanescent field of the mode penetrated more deeply into the environment. Thus, we decided to fabricate devices into the SQW wafer. In the device process, a PC slab was formed by using $e$-beam lithography, $\mathrm{HI}$ inductively coupled plasma etching, and the $\mathrm{HCl}$ selective wet etching of InP claddings.

In the measurement, the $\mathrm{H} 0$ nanolaser was photopumped by $\mathrm{cw}$ laser light at $\lambda=0.98 \mu \mathrm{m}$, which was focused to a $2.5-\mu \mathrm{m}$ spot on the top surface of the device through a $\times 50$ objective lens. The light output from the device was coupled to a multi-mode fiber by the same objective lens, and its emission spectrum was analyzed by using an optical spectrum analyzer (OSA). In this experiment, we deposited a chemically stable refractive index liquid (B-0700/0701, $n_{\text {env }}=1.296-1.451$ ) on the device. Figure 1(a) shows an example of the lasing spectrum of the $\mathrm{H} 0$ nanolaser obtained at room temperature $(293 \mathrm{~K})$ with $n_{\text {env }}=1.306$ and an irradiated power of $130 \mu \mathrm{W}$. The inset shows the scanning electron micrograph (SEM) of the

\#94556 - \$15.00 USD Received 4 Apr 2008; revised 10 May 2008; accepted 11 May 2008; published 20 May 2008 


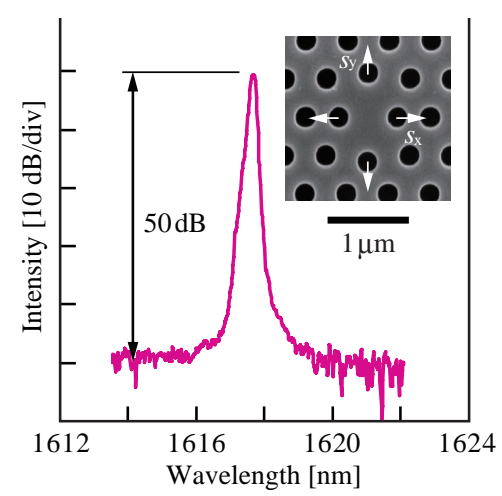

(a)

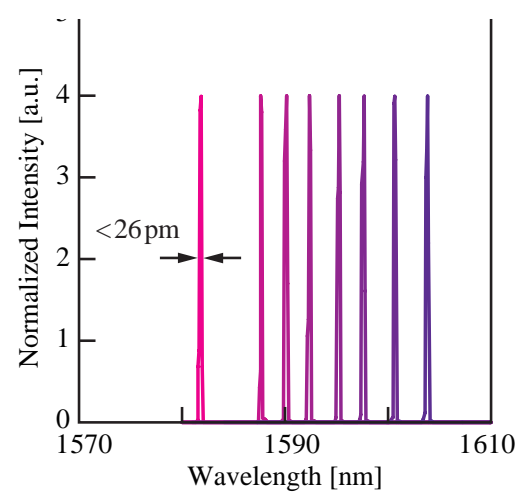

(b)

Fig. 1. Room-temperature cw lasing characteristics of $\mathrm{H} 0$ nanolaser in liquids. (a) Lasing spectrum and SEM image of the measured device. (b) Lasing spectra for $n_{\mathrm{env}}=1.296,1.315,1.325,1.335,1.344,1.354,1.363$, 1.373 from left to right.

measured device with $a=520 \mathrm{~nm}, 2 r=300 \mathrm{~nm}(2 r / a=0.58), s_{\mathrm{x}}=120 \mathrm{~nm}\left(s_{\mathrm{x}} / a=0.23\right)$ and $s_{\mathrm{y}}$ $=60 \mathrm{~nm}\left(s_{\mathrm{y}} / a=0.12\right)$. The three-dimensional finite-difference time-domain (FDTD) calculation showed that the $Q$ factor of the monopole mode for this design is higher than 4000,

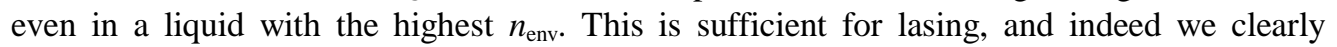
observed a cw lasing spectrum. The laser peak exhibited a 50-dB intensity over the background level and a spectral linewidth of $<26 \mathrm{pm}$, the resolution limit of the OSA used. The peak intensity was higher than in air [15]. We believe that this was attributable to the heat sinking by immersing the device in the liquid. Figure 1(b) shows a spectral redshift with $n_{\text {env }}$.

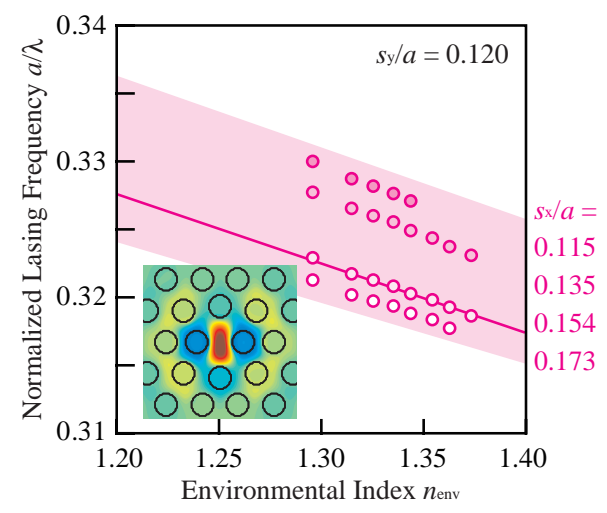

(a)

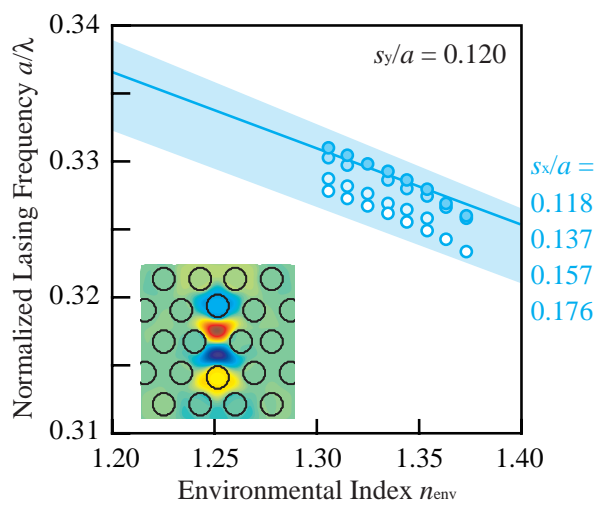

(b)

Fig. 2. Modal behaviors of HO nanolaser. Theoretical lines and experimental plots (open circles) of lasing wavelength for (a) monopole mode and (b) dipole mode. Each inset show calculated modal profile $\left(H_{\mathrm{z}}\right)$.

This simply indicates that we can use this shift for index sensing. The average index sensitivity in this measurement was $290 \mathrm{~nm} / \mathrm{RIU}$. On the basis of the spectral linewidth of < $26 \mathrm{pm}$, we can evaluate an index resolution of $9.0 \times 10^{-5}$. Note that $\mathrm{cw}$ microlasers, e.g., vertical-cavity surface-emitting lasers, usually exhibit a narrow frequency linewidth of less than $10 \mathrm{MHz}$ (a wavelength linewidth of less than $0.1 \mathrm{pm}$ ) [17]. Thus, a potential resolution of $<10^{-6}$ is expected.

Let us discuss the sensing characteristics in more detail. Figure 2 shows the normalized

\#94556 - \$15.00 USD Received 4 Apr 2008; revised 10 May 2008; accepted 11 May 2008; published 20 May 2008 


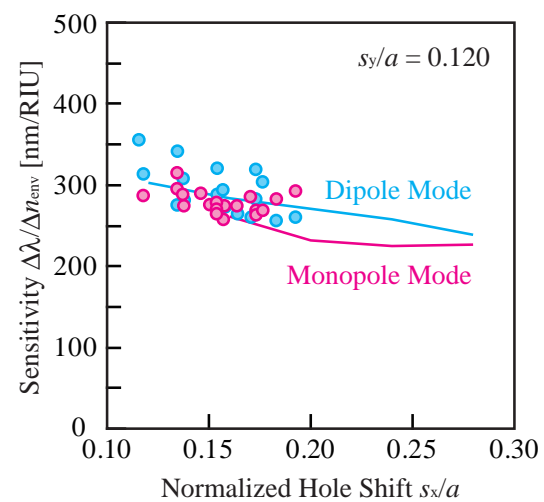

(a)

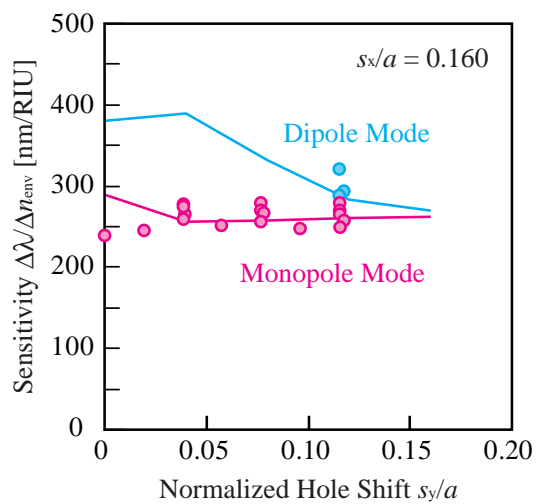

(b)

Fig. 3. Theoretical lines and experimental plots (open circles) of index sensitivity $\Delta \lambda / \Delta n_{\text {env }}$ with (a) $s_{\mathrm{x}}$ and (b) $s_{\mathrm{y}}$.

resonant frequency $a / \lambda$ with $n_{\text {env }}$ and their field distribution of the monopole and dipole modes. The solid line was calculated for $a=500 \mathrm{~nm}, 2 r=300 \mathrm{~nm}(2 r / a=0.60), d=160 \mathrm{~nm}, s_{\mathrm{x}}=80$ $\mathrm{nm}\left(s_{\mathrm{x}} / a=0.16\right)$ and $s_{\mathrm{y}}=60 \mathrm{~nm}\left(s_{\mathrm{y}} / a=0.12\right)$ by using the FDTD method. We assumed a uniform slab index of 3.4, neglecting the complicated layer structure of the actual epiwafer. In general, the dipole mode has a higher index sensitivity than the monopole mode because of the different penetration depths of the evanescent field into the environment. Circular plots show the experimental values for various normalized lattice shift $s_{\mathrm{x}} / a$. Corresponding well to the calculation, $a / \lambda$ shifts almost linearly with $n_{\text {env. }}$. The lasing wavelength could also be controlled by changing $s_{\mathrm{x}} / a$. Note that sensing using the nanolaser array is difficult if the sensitivity changes with $s_{\mathrm{x}}$ and $s_{\mathrm{y}}$. Figure 3 shows the calculated and measured index sensitivity $\Delta \lambda / \Delta n_{\mathrm{env}}$ as a function of $s_{\mathrm{x}}$ and $s_{\mathrm{y}}$. The sensitivity of the monopole mode is less dependent on $s_{\mathrm{x}}$ and $s_{\mathrm{y}}$ and ranges from 250 to $300 \mathrm{~nm} / \mathrm{RIU}$. No cw operation was obtained in the dipole mode for $s_{\mathrm{y}} / a<0.12$, which might be due to the low $Q$. At $s_{\mathrm{x}} / a=0.12$ and $s_{\mathrm{y}} / a=$ 0.12 , a sensitivity of $350 \mathrm{~nm} / \mathrm{RIU}$ was obtained for the dipole mode, and the estimated index resolution was $9.0 \times 10^{-5}$. This sensitivity is 1.4 times higher than the reported value [12]. This suggests that the field penetration of the dipole mode in air is particularly large. At $s_{\mathrm{y}} / a>0.12$ and $s_{\mathrm{y}} / a=0.12$, the sensitivity decreases with $s_{\mathrm{x}} / a$ and becomes almost comparable to that of the monopole mode. Although the high sensitivity of the dipole mode is attractive, the monopole mode is more advantageous for controlling the lasing wavelength by stabilizing the sensitivity. In addition, the $Q$ of the monopole mode is always higher than that of the dipole mode for any parameter change. Dipole-mode lasing is always accompanied by monopole-mode lasing. In other words, single-mode lasing by the monopole mode is obtained by setting $s_{\mathrm{y}} / a<0.12$. Thus, it is wise to use the monopole mode for stable sensing.

\section{Spectrometer-free sensing using nanolaser array}

Considering the field penetration from the nanocavity to the $\mathrm{PC}$ area, we roughly estimated that the $\mathrm{H} 0$ nanolaser occupies an effective area of $10 \times 10 \mu \mathrm{m}^{2}$. This means that we can fabricate one thousand nanolasers in a $340 \times 340 \mu \mathrm{m}$ area. If we fabricate a nanolaser array, in which each pair of lasing wavelengths is separated by $20 \mathrm{pm}$ and the NFP can be sharply discriminated by a BPF, it becomes a spectrometer-free sensor having an index resolution of $10^{-4}$. It could be challenging to control such a narrow wavelength separation to be uniform. However, although it is somewhat inhomogeneous, a similar sensing is obtained by matching the observed NFP and a reference pattern taken in advance for each device.

To demonstrate the concept, we fabricated an array of four nanolasers with $a=500 \mathrm{~nm}, 2 r$ $=280 \mathrm{~nm}(2 r / a=0.56), s_{\mathrm{x}}=60-90 \mathrm{~nm}\left(s_{\mathrm{x}} / a=0.12-0.18\right)$ and $s_{\mathrm{y}}=60 \mathrm{~nm}\left(s_{\mathrm{y}}=0.12\right)$, as 


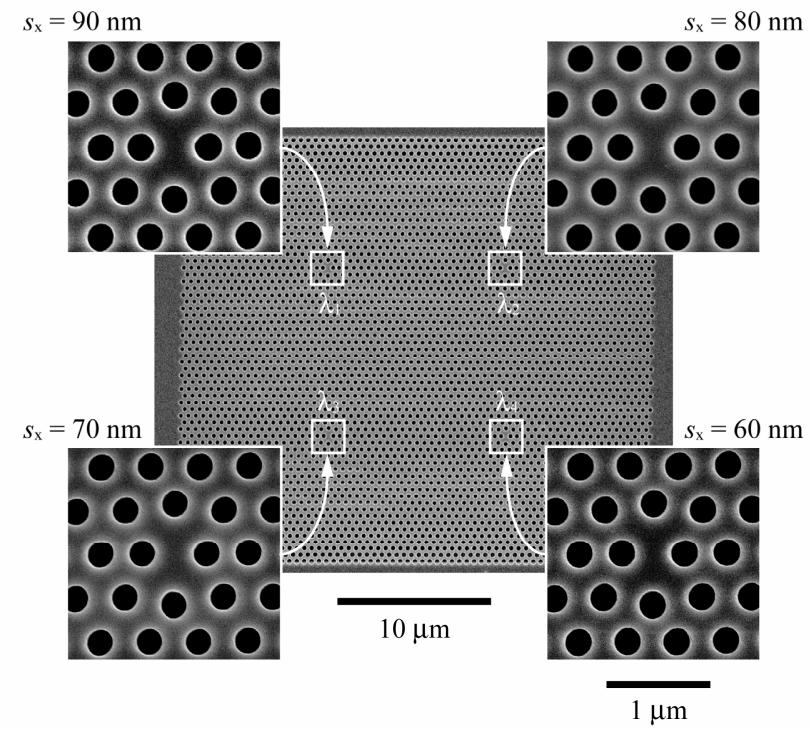

Fig. 4. SEM image of fabricated $2 \times 2 \mathrm{H} 0$ nanolaser array.

shown Fig. 4. Let us denote their lasing wavelengths by $\lambda_{1}, \lambda_{2}, \lambda_{3}$ and $\lambda_{4}$. The cavity spacing was set to $24 a(=11 \mu \mathrm{m})$ because we experimentally confirmed that the spacing was sufficiently large to suppress the mode coupling between cavities. But a smaller spacing might be possible by optimizing the cavity structure for reducing the field penetration and/or the cavity arrangement, taking into account the direction of the field penetration. In the measurement, we focused a laser light of $\lambda=0.98 \mu \mathrm{m}$ to a $25-\mu \mathrm{m}$ diameter spot and simultaneously photopumped all the devices. We observed the NFP by using an InGaAs image sensor through a BPF of multilayer dielectric stack (transmittance was $<10,50$, and $>90 \%$ at $\lambda=1.534,1.544$, and $1.552 \mu \mathrm{m}$, respectively). Figure 5 shows the lasing spectra and the corresponding NFPs for different $n_{\text {env }}$. We confirmed during the individual pumping of each device that the lasing wavelengths were ordered as designed. The index sensitivity was $\sim 300$ $\mathrm{nm} / \mathrm{RIU}$ and almost constant for all the lasers. The wavelength separation was inhomogeneous in the $2-10 \mathrm{~nm}$ range, and partial multimode lasing was observed. The multimode lasing was caused by the $s_{\mathrm{y}}$ in this preliminary experiment; it was employed to ensure a high $Q$ of the monopole mode for all the devices. If the fabrication process is improved so that the uniform lasing by the monopole mode is obtained more easily for all the devices even in the liquids, a smaller $s_{\mathrm{y}}$ is more desirable for the complete suppression of the dipole mode. But anyway, we could clearly observe the target operation in the NFPs; the number of laser spots did not change without the BPF, but changed with BPF due to the wavelength shift.

\section{Conclusions}

We obtained clear cw operation in a H0 PC nanolaser soaked in liquids whose refractive index ranged from 1.00 to 1.37 . The maximum intensity of the laser mode was $50 \mathrm{~dB}$ higher than the background level, and the spectral linewidth was $<26 \mathrm{pm}$, which is the resolution limit of this measurement. We observed a wavelength shift with the environmental index. The maximum sensitivity was $350 \mathrm{~nm} / \mathrm{RIU}$, the highest value recorded for nanocavity-based index sensing. Thus, we confirmed an index resolution of $9.0 \times 10^{-5}$. But we expect to be able to obtain a resolution of $<10^{-6}$, given that the linewidth in microlasers is usually on the order of $0.1 \mathrm{pm}$. This will be checked in future studies using heterodyne detection. We also demonstrated index 
sensing using a nanolaser array consisting of different wavelength devices. This allows spectrometer-free sensing and potentially a $10^{-4}$-order resolution.

As these sensors do not require a wideband light source, high-precision optical I/O and spectrometer, it will be useful for low-cost and disposal biochemical sensing and label-free single molecular sensing. Also, it is expected that they will be integrated as a functional element in the lab-on-a-chip technology.

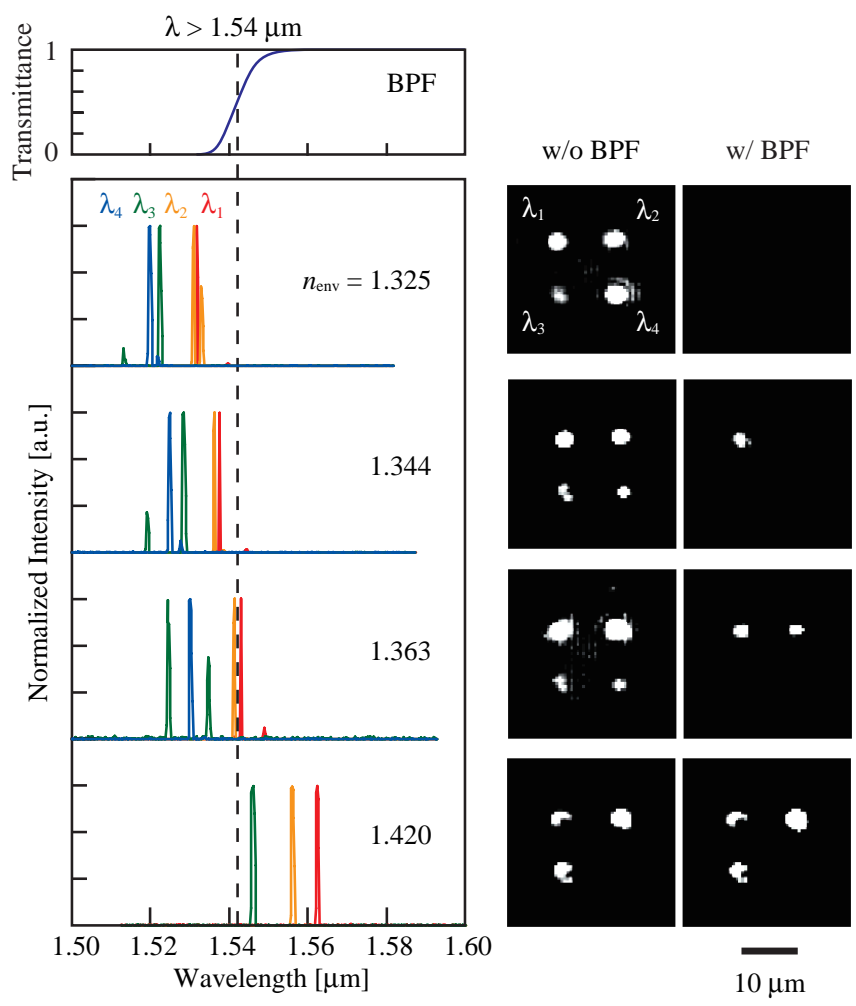

Fig. 5. Lasing spectra and NFP of nanolaser array for different $n_{\text {env }}$. Transmission spectrum of BPF used for the observation of the NFP is shown above the spectra. NFPs. 